\title{
Los procesos de gestión de documentos en la Ley General de Archivos de México: análisis de contenido desde una perspectiva cuantitativa
}

\author{
José-Luis Bonal-Zazo* \\ María-Pilar Ortego-de-Lorenzo-Cáceres*
}

Artículo recibido:

10 de diciembre de 2019

Artículo aceptado:

30 de marzo de 2020

Artículo de investigación

\section{Resumen}

Se analizan las referencias sobre procesos de creación, captura y gestión de documentos en la Ley General de Archivos (LGA) de México mediante una metodología cuantitativa de análisis de contenido, la cual permite valorar, tanto de forma absoluta como ponderada, la presencia de seis grandes categorías de procesos en la LGA: Valoración y disposición; Conservación y preservación; Organización y descripción; Acceso y transparencia; Difusión, y, por último, Producción y seguimiento de documentos; así como el enfoque bajo el que es tratada cada una de las categorías (actividades, agentes o

* Universidad de Extremadura, Facultad de Ciencias de la Documentación y la Comunicación, España

jlbonal@unex.es_portego@unex.es

INVESTIGACIÓN BIBLIOTECOLÓGICA, vol. 34, núm. 85, octubre/diciembre, 2020, México, ISSN: 2448-8321 pp. 179-197 
elementos). Mediante la metodología apuntada, se han identificado 347 referencias sobre las categorías señaladas, con un valor ponderado global de 301 puntos, la mayoría de los cuales corresponden a las categorías de Valoración y disposición (29.40%); Conservación y preservación (26.58 \%); Organización y descripción (18.44 \%), y Acceso y transparencia (15.94\%); mientras que las dos categorías restantes presentan valores inferiores a $6 \%$. El análisis de los resultados permite concluir que en la LGA se conjugan procesos archivísticos tradicionales con procesos más novedosos, como trazabilidad, interoperabilidad, seguridad de información o gestión de metadatos.

Palabras clave: Ley General de Archivos; Procesos de Gestión Documental; Análisis de contenido; México

The records management of processes in the General Archives Law of México: content analysis from a quantitative perspective José-Luis Bonal-Zazo and María-Pilar Ortego-de-Lorenzo-Cáceres

\section{Abstract}

The references on processes of creation, capture and management of documents in the General Law of Archives (LGA) of Mexico are analysed through a quantitative methodology of content analysis, which allows assessing, both in absolute and weighted form, the presence of six large process categories in the LGA: appraisal and disposition; conservation and preservation; organization and description; access and transparency; diffusion; and, finally, production and traceability of documents; as well as the approach under which each of the categories (activities, agents or elements) is treated. Through the methodology indicated, 347 references have been identified on the indicated categories, with a global weighted value of 301 points, most of which correspond to the categories of appraisal and disposition (29.40\%); conservation and preservation $(26.58 \%)$; organization and description (18.44\%); and access and transparency (15.94\%); while the remaining two categories have values below 
$6 \%$. The analysis of the results allows us to conclude that in the LGA traditional archival processes are combined with more innovative processes, such as traceability, interoperability, information security or metadata management.

Keywords: General Archives Law; Records Management Processes; Content Analysis; Mexico

\section{INTRODUCCIÓN}

S egún la norma UNE-ISO 15489-1, un "proceso de trabajo" ("work process") Ses "una o más secuencias de operaciones que se requieren para producir un resultado y que está sujeto a unas determinadas reglas o procedimientos" (UNE-ISO 15489-1, 2016: 9). Un proceso, por tanto, se define por tres rasgos: tiene un carácter compuesto, ya que está formado por un conjunto de operaciones o acciones; siempre produce un resultado final, un resultado que puede ser la elaboración de un producto o la prestación de un servicio, y, por último, tiene un carácter normalizado, ya que debe atenerse a determinadas reglas o procedimientos.

La importancia de los procesos es tal que, según la norma ISO 9001 (2015), uno de los siete principios de la gestión de calidad es el "enfoque a procesos". Se trata de un principio según el cual la gestión de las actividades de una organización es más eficiente si se sustenta sobre procesos bien definidos para alcanzar los resultados previstos. "Enfocar" la gestión hacia los procesos conlleva, entre otras, las siguientes exigencias: la identificación de los procesos, el conocimiento de sus elementos, la definición sistemática de la secuencia de acciones de cada proceso, la identificación de relaciones con otros procesos, el establecimiento de responsabilidades y la aplicación de mecanismos de medición y mejora; aspectos estudiados con detalle por Pérez Fernández de Velasco (2007).

Los archivos, como organizaciones responsables de los documentos, también pueden gestionar sus actividades de trabajo como procesos siguiendo las directrices de la ISO 9001, porque, tal como señala Zaratiegui (1999), es preciso pasar "de la gestión de los procesos a la gestión por procesos". En este sentido, la norma UNE-ISO 15489-1 (2016) identifica ocho procesos relacionados con la creación, captura y gestión de documentos: 1) creación, 2) captura, 3) clasificación e indización, 4) control de acceso, 5) almacenamiento, 6) uso y reutilización, 7) migración y conversión, y 8) disposición. 
No obstante, el análisis de los procesos de trabajo en los archivos ha sido abordado en diferentes trabajos a lo largo del tiempo. Autores como Gallardo Taboada (1987), Evans (1990), Barnard Amozorrutia (2011), Sainz Varela (2011), Daines (2011), Bustelo Ruesta (2011) o Llansó Sanjuan (2012) se han ocupado del tema desde diferentes perspectivas teóricas. En otros casos, autores como Villanueva Bazán et al. (2002), Bustelo Ruesta (2017) o Bustos Pretel (2018) han definido, desde el punto de vista técnico, los procesos de gestión documental.

En el contexto descrito, la publicación de la Ley General de Archivos de México en 2018, y su entrada en vigor el 15 de junio de 2019, constituye un hito relevante en la archivística mexicana que, por otra parte, se enmarca en lo que Aguilera Murguía (2017) denomina "un nuevo entorno de valores”, caracterizado por la aparición de una extensa normativa sobre acceso a la información, transparencia, rendición de cuentas, anticorrupción y gobierno abierto.

La Ley General de Archivos (en adelante LGA) se encuentra organizada en tres libros, dedicados, respectivamente, a la organización y administración de los archivos, al Archivo General de la Nación, y a las infracciones y delitos en materia de archivos. Aunque se trata de una fuente eminentemente legal, también se ocupa de cuestiones técnicas y, por esa razón, resulta relevante conocer el enfoque de la Ley sobre los procesos relacionados con la producción y el tratamiento documental.

Por todo lo expuesto, este trabajo se plantea con el objetivo general de conocer cómo son tratados los procesos relacionados con la creación, captura y gestión de documentos en la LGA. De manera más precisa, se formulan los siguientes objetivos específicos:

1. Analizar de forma cuantitativa la presencia de los procesos generales y los procesos específicos relacionados con la creación, captura y gestión de documentos en la LGA.

2. Analizar, de forma cuantitativa, los enfoques o facetas bajo los cuales se abordan los procesos identificados.

\section{Metodología}

Para cumplir los objetivos propuestos se ha diseñado una metodología basada en el uso de técnicas de análisis de contenido. El análisis de contenido permite conocer la forma en que es tratado un tema en un documento o en un conjunto de documentos de manera objetiva, sistemática y cuantitativa (Wimmer y Dominick, 1996: 170-171). El trabajo se ha desarrollado en cinco fases. 
En una fase inicial se llevó a cabo la definición y determinación del alcance del tema. Teniendo en cuenta los objetivos definidos, se estableció que el tema objeto de estudio debían ser los procesos relacionados con la creación, uso y gestión de documentos, así como los instrumentos resultantes de la aplicación de los mismos, los cuales constituyen un reflejo de los procesos que dan lugar a su origen (clasificación, disposición, consulta...).

La segunda fase consistió en la definición de las unidades de análisis. Las unidades de análisis, o unidades de registro, son los elementos que deben ser cuantificados (Wimmer y Dominick, 1996: 178). Tal como señala Bardin (1991: 78-81), estas unidades pueden ser de diferente tipo: palabras, temas, objetos, acontecimientos, etc. En nuestro caso, optamos por definir como unidad de análisis cada enunciado expresado en la LGA sobre procesos e instrumentos de gestión de documentos con significación plena. Se han identificado varios tipos de enunciados:

- Enunciados completos individuales. Es decir, enunciados válidos por sí mismos de forma independiente. Por ejemplo, el artículo 47 de la LGA establece que "los sujetos obligados conservarán los documentos de archivo aun cuando hayan sido digitalizados".

- Enunciados inteligibles en un contexto determinado. Se trata de enunciados que deben ser contextualizados para que adquieran significación plena. Por ejemplo, en el artículo 31 de la LGA se encuentra la siguiente disposición: "realizar las transferencias primarias al archivo de concentración", la cual alude a una de las funciones de los archivos de trámite; por tanto, la idea completa que subyace en el enunciado es "[los archivos de trámite deben] realizar las transferencias primarias al archivo de concentración".

- Enunciados compuestos. Frases compuestas que incluyen varias ideas diferentes. Por ejemplo, el artículo 40 de la LGA establece que "los responsables de los archivos históricos de los sujetos obligados, adoptarán medidas para fomentar la preservación y difusión de los documentos con valor histórico"; en este enunciado se hace referencia a dos procesos diferentes: preservación y difusión.

- Enumeraciones. Frases que incluyen una enumeración de procesos. Por ejemplo, el artículo 105 de la Ley señala que el Archivo General de la Nación debe "desarrollar investigaciones encaminadas a la organización, conservación y difusión del patrimonio documental que resguarda". Como se puede advertir, se alude a tres procesos diferentes: organización, conservación y difusión. 
Teniendo en cuenta los criterios indicados, fueron identificadas en la LGA 347 referencias diferentes. Una vez definidas las unidades de análisis se procedió, en tercer lugar, a la definición de las categorías de contenido, para lo cual se siguieron las directrices de la norma UNE-ISO 30301 (2011) y de la norma UNE-ISO 15489-1 (2016: 23-27), las cuales describen los procesos necesarios para la creación, captura y gestión de documentos. Tras una lectura detallada de ambas normas y de la Ley se establecieron seis grandes categorías de contenido, correspondientes a seis grandes procesos o grupos de procesos de gestión de documentos. A su vez, dentro de cada una de ellas se ha establecido un número variable de subcategorías (Tabla 1), enlistadas a continuación.

- Producción y seguimiento. Procesos relacionados con la gestión de los documentos en sus primeras fases. Se han definido tres subcategorías: Producción y recepción, Seguimiento (o trazabilidad), e Intercambio (o interoperabilidad).

- Organización y descripción. Ambos procesos son tratados de forma conjunta en la LGA, donde se define "organización" como el "conjunto de operaciones intelectuales y mecánicas destinadas a la clasificación, ordenación y descripción de los distintos grupos documentales" (LGA, art. 4, XLII). Dentro de esta categoría se han diferenciado cuatro subcategorías: Fundamentos generales de organización (clasificación + ordenación + descripción), Elaboración de cuadros de clasificación, Elaboración de instrumentos de consulta, y Descripción/gestión de metadatos.

- Valoración y disposición. Como en el caso anterior, se trata de dos procesos diferentes, pero vinculados entre sí, que a menudo se presentan juntos. Se han establecido tres subcategorías: Valoración, Disposición, y Elaboración del catálogo de disposición documental.

- Conservación y preservación. Se trata de una categoría dedicada a la conservación y preservación de documentos analógicos y digitales. Se han establecido tres subcategorías: Fundamentos de conservación y preservación, Preservación digital, y Protección legal de los documentos.

- Acceso y transparencia. Categoría relativa al binomio acceso a la información/protección de la información. Se han establecido tres subcategorías: Acceso, Transparencia, y Seguridad de la información.

- Difusión. Difusión y divulgación del patrimonio documental. Se han definido dos subcategorías: Difusión de documentos y Difusión de archivos. 
Cada una de las categorías y subcategorías enunciadas puede ser tratada desde diferentes puntos de vista o facetas. Por ejemplo, la valoración y disposición documental son procesos en los que pueden estar implicados diversos agentes (sujetos obligados, servidores públicos, áreas productoras...), que se encuentran integrados por varias actividades (valoración, disposición, baja, transferencia...) y en los cuales se utilizan o generan diferentes elementos (ficha técnica de valoración, catálogo de disposición...). Por esa razón, teniendo en cuenta la naturaleza de los procesos analizados, se definieron, de forma complementaria, tres facetas (Tabla 1):

- Agentes: personas físicas e instituciones implicadas en el desarrollo de los procesos.

- Actividades: acciones o conjuntos de acciones que forman parte de los procesos o están relacionados con ellos.

- Elementos: componentes de los procesos; bien de carácter concreto o tangible (inventario, cuadro de clasificación...), bien de carácter abstracto o intangible (vigencia documental, ciclo vital...).

\begin{tabular}{|c|c|c|c|c|c|c|}
\hline Tipos & \multicolumn{6}{|c|}{ Categorías de contenido definidas } \\
\hline Categorías & $\begin{array}{c}\text { Producción } \\
\text { y seguimiento }\end{array}$ & $\begin{array}{l}\text { Organización } \\
\text { y descripción }\end{array}$ & $\begin{array}{l}\text { Valoración y } \\
\text { disposición }\end{array}$ & $\begin{array}{l}\text { Conservación y } \\
\text { preservación }\end{array}$ & $\begin{array}{c}\text { Acceso } \\
\text { y transparencia }\end{array}$ & Difusión \\
\hline \multirow{4}{*}{ Subcategorías } & $\begin{array}{l}\text { Producción } \\
\text { y recepción }\end{array}$ & $\begin{array}{l}\text { Fundamentos } \\
\text { de } \\
\text { organización }\end{array}$ & Valoración & $\begin{array}{l}\text { Fundamentos de } \\
\text { conservación y } \\
\text { preservación }\end{array}$ & Acceso & $\begin{array}{l}\text { Difusión de } \\
\text { documentos }\end{array}$ \\
\hline & $\begin{array}{l}\text { Seguimiento } \\
\text { (trazabilidad) }\end{array}$ & $\begin{array}{l}\text { Elaboración } \\
\text { del cuadro de } \\
\text { clasificación }\end{array}$ & Disposición & $\begin{array}{l}\text { Preservación } \\
\text { digital }\end{array}$ & Transparencia & \multirow{3}{*}{$\begin{array}{c}\text { Difusión } \\
\text { de archivos }\end{array}$} \\
\hline & \multirow{2}{*}{$\begin{array}{c}\text { Intercambio } \\
\text { (interoperabilidad) }\end{array}$} & $\begin{array}{l}\text { Elaboración de } \\
\text { instrumentos } \\
\text { de consulta }\end{array}$ & \multirow{2}{*}{$\begin{array}{c}\text { Elaboración } \\
\text { del catálogo } \\
\text { de } \\
\text { disposición }\end{array}$} & \multirow[t]{2}{*}{ Protección legal } & \multirow{2}{*}{$\begin{array}{l}\text { Seguridad } \\
\text { de la } \\
\text { información }\end{array}$} & \\
\hline & & $\begin{array}{l}\text { Descripción/ } \\
\text { gestión de } \\
\text { metadatos }\end{array}$ & & & & \\
\hline \multirow{3}{*}{ Facetas } & \multicolumn{6}{|c|}{ Agentes } \\
\hline & \multicolumn{6}{|c|}{ Actividades } \\
\hline & \multicolumn{6}{|c|}{ Elementos } \\
\hline
\end{tabular}

Tabla 1. Categorías de contenido definidas (categorías, subcategorías y facetas) 
Cada una de las 347 referencias identificadas en la fase anterior fue clasificada en alguna de las categorías señaladas en la Tabla 1.

La cuarta fase consistió en la determinación del sistema de cuantificación. Las referencias sobre procesos identificadas en la LGA son, con carácter general, de dos tipos: referencias breves, que contienen disposiciones genéricas, indirectas o simples enumeraciones, y referencias extensas, que presentan disposiciones específicas, contenidos detallados y definiciones directamente relacionadas con los procesos. Teniendo en cuenta el carácter del texto de las disposiciones, se estimó que el sistema más objetivo para valorar cuantitativamente las referencias seleccionadas es la frecuencia ponderada (Bardin, 1991: 82-87). Para ello se aplicó el siguiente factor de ponderación: $\mathrm{N}^{*} 0.5$ para referencias breves y $\mathrm{N}^{*} 1$ para referencias extensas. De este modo, es posible calcular el peso de cada proceso en la Ley mediante el siguiente cálculo:

$$
\left(\mathrm{Nrb}^{*} 0.5\right)+(\mathrm{Nre} * 1)
$$

donde:

$\mathrm{Nrb}=$ número de referencias breves

Nre $=$ número de referencias extensas

$0.5=$ factor de ponderación para referencias breves

$1=$ factor de ponderación para referencias extensas

La aplicación del factor de ponderación a cada una de las 347 referencias seleccionadas dio lugar a la obtención de un valor ponderado final global de 301, ya que se identificaron 255 referencias extensas que mantuvieron su valor porque se aplicó el factor de ponderación de 1, y 92 referencias breves, que fueron multiplicadas por el factor 0.5 , de tal manera que su peso se redujo a la mitad.

La quinta y última fase se dedicó a la codificación del contenido y la recogida de datos mediante una plantilla integrada por ocho campos (número de referencia, texto seleccionado de la LGA, factor de ponderación, categoría, subcategoría, faceta, resumen, y observaciones) que permitió recoger la información de manera sistemática y cuantificar los datos. 


\section{Presentación y ANÁlisis DE RESUltados}

\section{Distribución por categorías y subcategorias}

Como ya hemos señalado, se identificaron 347 referencias relativas a procesos de gestión documental: 255 extensas y 92 breves.

Existen dos categorías que predominan sobre el resto: se trata de Valoración y disposición, con un porcentaje del valor ponderado de $29.40 \%$, y Conservación y preservación, con un porcentaje del mismo valor de $26.58 \%$. Cabe destacar el hecho de que en la categoría Valoración y disposición el porcentaje sobre el valor ponderado es más elevado $(29.40 \% ; n=88.5, \mathrm{~N}=301)$ que el porcentaje sobre el valor absoluto (número total de referencias) (27.38 \%; $\mathrm{n}=95, \mathrm{~N}=347)$, lo cual indica que la mayoría de referencias sobre este proceso son referencias extensas. Por el contrario, la categoría Conservación y preservación presenta unos porcentajes similares en el valor absoluto ( $26.51 \% ; n=92, N=347)$ y en el valor ponderado $(26.58 \% ; n=80, N=301)$.

En un nivel intermedio se encuentran las categorías de Organización y descripción (18.44 \%) y Acceso y transparencia (15.94 \%). En el primer caso el porcentaje sobre el valor ponderado $(18.44 \% ; \mathrm{n}=55.5, \mathrm{~N}=301)$ es inferior al porcentaje sobre el valor absoluto $(20.46 \% ; n=71, N=347)$, lo que significa que existe un elevado número de referencias breves sobre el tema. En cambio, en Acceso y transparencia el porcentaje sobre el valor absoluto (15.57\%) es similar al porcentaje sobre el valor ponderado (15.94\%), lo que supone la presencia de pocas referencias breves.

Las categorías temáticas con los porcentajes sobre el valor ponderado más bajos son Difusión (5.65 \%) y Producción y seguimiento (3.99 \%). En ambos casos los porcentajes sobre los valores absolutos y sobre los valores ponderados son parecidos (Tabla 2).

El análisis detallado de cada categoría permite advertir que existen diferencias significativas en la distribución de las subcategorías.

En la categoría Valoración y disposición, la distribución de referencias por subcategorías es uniforme, los valores ponderados de las tres subcategorías, aunque son diferentes, se encuentran próximos entre sí: Valoración (11.46 \%); Disposición (9.63 \%) y Elaboración del catálogo de disposición documental $(8.31 \%)$. Algo similar ocurre con la categoría Difusión, donde las dos subcategorías existentes presentan valores similares: Difusión de documentos $(2.99 \%)$ y Difusión de archivos $(2.66 \%)$.

Sin embargo, en las cuatro categorías restantes la distribución por subcategorías no es tan homogénea como en las dos anteriores. 


\begin{tabular}{|c|c|c|c|c|c|c|c|c|c|c|c|c|c|c|}
\hline \multirow{2}{*}{ 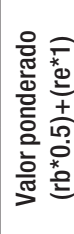 } & $\alpha^{\circ}$ & $\begin{array}{l}\text { \&े } \\
\text { N }\end{array}$ & $\begin{array}{l}\stackrel{Q}{q} \\
F\end{array}$ & ़ְ & $\begin{array}{c}\bar{\infty} \\
\infty\end{array}$ & $\begin{array}{l}\infty \\
\stackrel{\infty}{\infty} \\
\stackrel{N}{N}\end{array}$ & 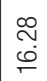 & లె & $\begin{array}{l}\text { बे } \\
\text { ल) }\end{array}$ & $\begin{array}{l}\stackrel{+}{+} \\
\infty\end{array}$ & ल & $\underset{\omega}{\bar{\sigma}}$ & ঙ্ণী & مִ \\
\hline & 竞 & $\begin{array}{l}\text { L } \\
\infty \\
\infty\end{array}$ & 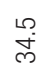 & R & $\stackrel{\llcorner}{\sim}$ & ○ & 守 & $\stackrel{\square}{\square}$ & $\simeq$ & 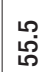 & $\approx$ & $\stackrel{\circ}{\circ}$ & $\simeq$ & مْ \\
\hline \multirow{2}{*}{ 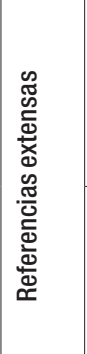 } & 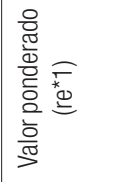 & ळ & m & $\stackrel{d}{\sim}$ & $\stackrel{\llcorner}{\sim}$ & œ & $\hat{m}$ & $\stackrel{9}{\square}$ & $\simeq$ & \& & 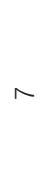 & $\stackrel{\circ}{\circ}$ & $\simeq$ & ما \\
\hline & 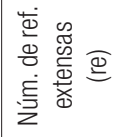 & ळ & $\stackrel{m}{m}$ & $\stackrel{d}{\sim}$ & $\stackrel{\llcorner}{\sim}$ & ஜ & $\hat{m}$ & $\stackrel{\sigma}{\square}$ & $\simeq$ & 우 & 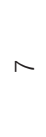 & $\stackrel{0}{\sim}$ & $\simeq$ & مـ \\
\hline
\end{tabular}

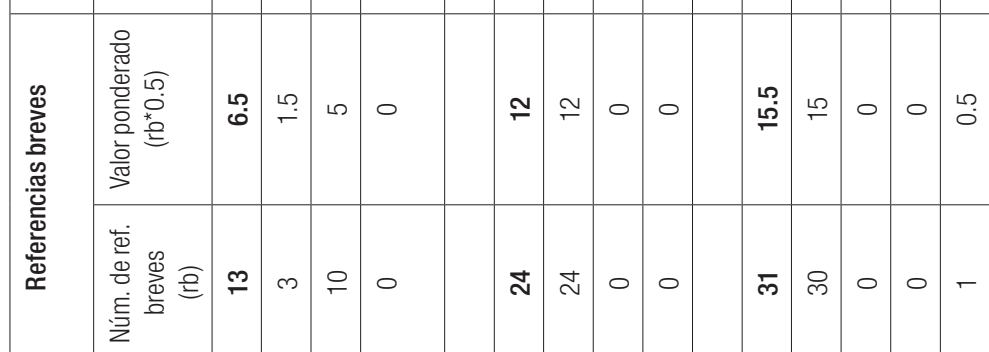

\begin{tabular}{|c|c|c|c|c|c|c|c|c|c|c|c|c|c|c|}
\hline 일 & $\circ^{\circ}$ & 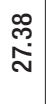 & $\begin{array}{l}\infty \\
\text { లె } \\
\stackrel{0}{0}\end{array}$ & $\begin{array}{l}\infty \\
\infty \\
\infty\end{array}$ & 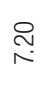 & $\begin{array}{l}\overline{5} \\
\stackrel{0}{0}\end{array}$ & 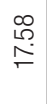 & 告 & 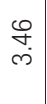 & \&̊+ & $\begin{array}{l}0 \\
\stackrel{0}{0} \\
\stackrel{0}{0}\end{array}$ & $\underset{\forall}{\sigma}$ & $\begin{array}{l}\stackrel{q}{*} \\
\dot{m}\end{array}$ & $\stackrel{\mathscr{R}}{\stackrel{2}{\leftarrow}}$ \\
\hline $\begin{array}{l}\frac{0}{\pi} \\
\stackrel{0}{\circ}\end{array}$ & 容 & டூ & p) & ले & $\stackrel{2}{\sim}$ & ชี & $\bar{\sigma}$ & $\stackrel{\sigma}{\circ}$ & $\simeq$ & $\kappa$ & $\hat{m}$ & $\stackrel{\circ}{\circ}$ & $\simeq$ & \\
\hline
\end{tabular}

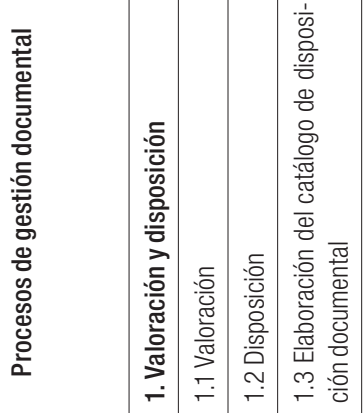

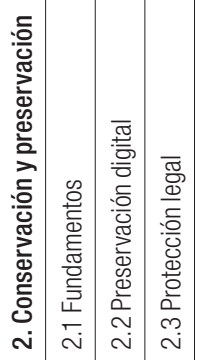

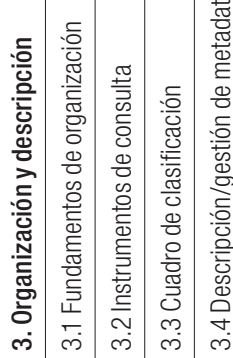




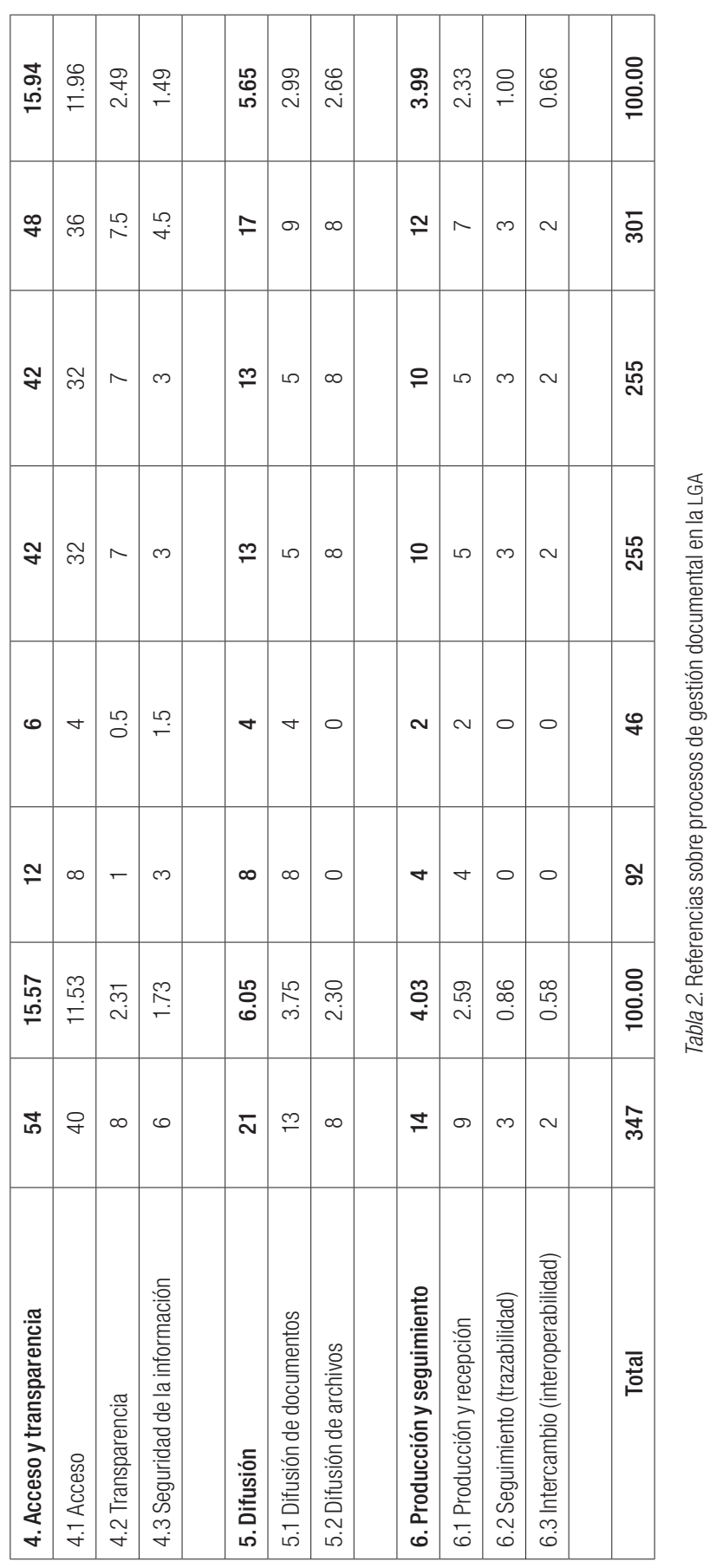


En el caso de la categoría Conservación y preservación, existe una diferencia significativa entre el porcentaje sobre el valor ponderado de la subcategoría Fundamentos de conservación y preservación (16.28 \%) frente a las otras dos subcategorías: Preservación digital (6.31 \%) y Protección legal (3.99 \%). Tal como se puede advertir, buena parte de las referencias identificadas (61 sobre un total de $92 ; 66.3 \%$ ) son referencias sobre fundamentos de la conservación y la preservación (se trata de la subcategoría con un mayor número de referencias); sin embargo, muchas de ellas son referencias breves, que apenas presentan información específica. Por el contrario, en el caso de las subcategorías Preservación digital y Protección legal, todas las referencias identificadas son, de acuerdo a los criterios descritos en la cuarta fase de la metodología, referencias extensas.

Algo similar ocurre en la categoría Organización y descripción, donde la subcategoría que presenta mayores resultados es Fundamentos de organización $(7.31 \%)$, seguida, con valores progresivamente inferiores, de las categorías Instrumentos de consulta (5.31\%), Cuadro de clasificación (3.99 \%), y Descripción/gestión de metadatos (1.83 \%). Pese a estos valores, la mayoría de las referencias de la subcategoría Fundamentos de organización son referencias breves (30 de 37; 81.08\%); en cambio, prácticamente todas las referencias del resto de subcategorías son referencias extensas, según la clasificación establecida en la cuarta fase de la metodología.

En la categoría Acceso y transparencia se advierte que la subcategoría Acceso predomina sobre el resto, con $11.96 \%$; se trata de la segunda subcategoría con un porcentaje más elevado en todo el conjunto. Las otras dos subcategorías presentan valores muy inferiores: Transparencia, $2.49 \%$, y Seguridad de la información, $1.49 \%$. La mayoría de las referencias incluidas en las tres subcategorías son referencias extensas.

La última categoría, Producción y seguimiento, presenta subcategorías con valores muy bajos y, proporcionalmente, diferentes entre sí: Producción y recepción (2.33\%); Seguimiento (trazabilidad) (1 \%) e Intercambio (interoperabilidad) $(0.66 \%)$. La mayoría de las referencias son de carácter extenso (Tabla 2). 


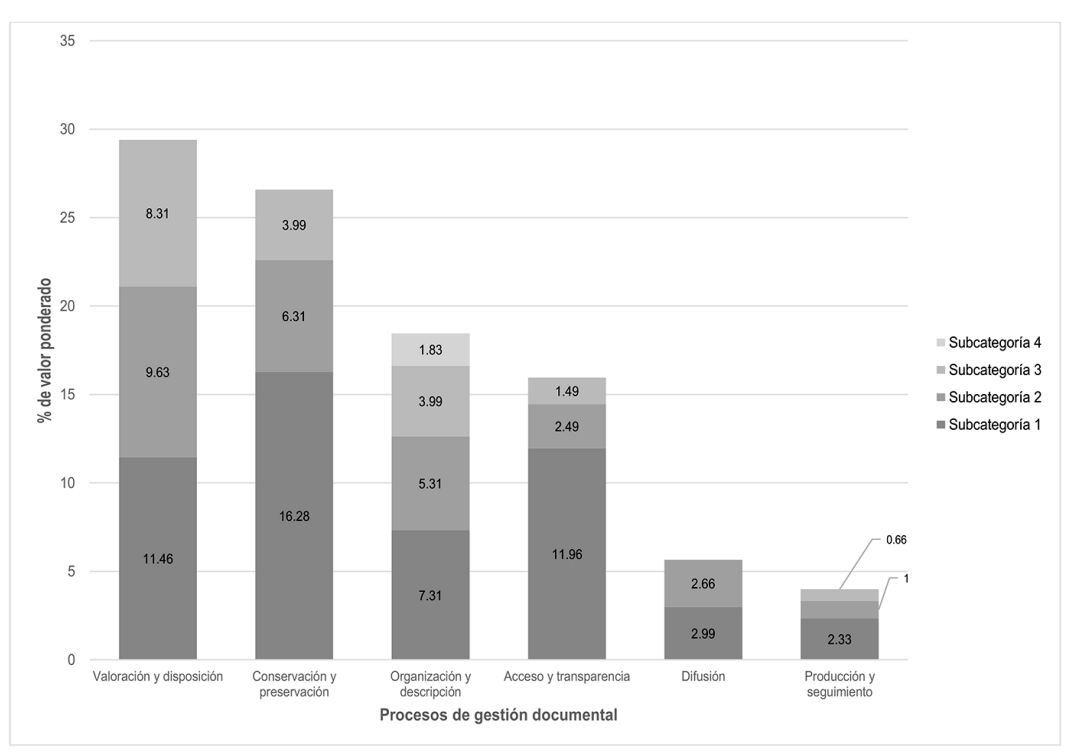

Figura 1. Procesos de gestión documental en la LGA ${ }^{1}$

\section{Distribución por facetas}

Todos los procesos de gestión documental analizados son tratados desde tres facetas diferentes: Actividades, Agentes y Elementos.

La faceta que presenta el porcentaje sobre el valor ponderado más elevado es Agentes con $67.27 \%$, lo que supone que la mayoría de las referencias identificadas son referencias relativas a funciones, competencias u obligaciones de los agentes implicados en la aplicación de la Ley, los cuales abarcan un amplio espectro: sujetos obligados, servidores públicos, jefes de misiones diplomáticas o consulares, grupos interdisciplinarios, áreas productoras, áreas coordinadoras, áreas operativas, archivos generales, archivos de concentración, archivos históricos, archivos de trámite, Sistema Nacional de Archivos, Sistema Nacional de Transparencia, Consejo Nacional de Archivos, Consejos Locales, AGN, IFAI, Alcaldía de la Ciudad de México o Estado mexicano. 


\begin{tabular}{|c|c|c|c|c|c|}
\hline \multirow{2}{*}{$\begin{array}{l}\text { Procesos } \\
\text { de gestión } \\
\text { documental }\end{array}$} & \multirow[t]{2}{*}{ Valores } & \multicolumn{3}{|c|}{ Facetas } & \multirow[t]{2}{*}{ Total } \\
\hline & & Actividades & Agentes & Elementos & \\
\hline \multirow{4}{*}{$\begin{array}{l}\text { 1. Valoración } \\
\text { y disposición }\end{array}$} & Núm. absoluto & 16 & 68 & 11 & 95 \\
\hline & $\%$ absoluto & 4.61 & 19.60 & 3.17 & 27.38 \\
\hline & Núm. ponderado & 15 & 63 & 10.5 & 88.5 \\
\hline & $\%$ ponderado & 4.98 & 20.93 & 3.49 & 29.40 \\
\hline \multirow{4}{*}{$\begin{array}{l}\text { 2. Conservación } \\
\text { y preservación }\end{array}$} & Núm. absoluto & 22 & 55 & 15 & 92 \\
\hline & $\%$ absoluto & 6.34 & 15.85 & 4.32 & 26.51 \\
\hline & Núm. ponderado & 18.5 & 47 & 14.5 & 80 \\
\hline & $\%$ ponderado & 6.15 & 15.61 & 4.82 & 26.58 \\
\hline \multirow{4}{*}{$\begin{array}{l}\text { 3. Organización } \\
\text { y descripción }\end{array}$} & Núm. absoluto & 10 & 53 & 8 & 71 \\
\hline & $\%$ absoluto & 2.88 & 15.27 & 2.31 & 20.46 \\
\hline & Núm. ponderado & 14 & 34.5 & 7 & 55.5 \\
\hline & $\%$ ponderado & 4.65 & 11.46 & 2.33 & 18.44 \\
\hline \multirow{4}{*}{$\begin{array}{l}\text { 4. Acceso } \\
\text { y transparencia }\end{array}$} & Núm. absoluto & 8 & 39 & 7 & 54 \\
\hline & $\%$ absoluto & 2.31 & 11.24 & 2.02 & 15.57 \\
\hline & Núm. ponderado & 6 & 36 & 6 & 48 \\
\hline & $\%$ ponderado & 1.99 & 11.96 & 1.99 & 15.94 \\
\hline \multirow[t]{4}{*}{ 5. Difusión } & Núm. absoluto & 3 & 18 & 0 & 21 \\
\hline & $\%$ absoluto & 0.86 & 5.19 & 0.00 & 6.05 \\
\hline & Núm. ponderado & 2 & 15 & 0 & 17 \\
\hline & $\%$ ponderado & 0.67 & 4.98 & 0 & 5.65 \\
\hline \multirow{4}{*}{$\begin{array}{l}\text { 6. Producción } \\
\text { y seguimiento }\end{array}$} & Núm. absoluto & 6 & 8 & 0 & 14 \\
\hline & $\%$ absoluto & 1.72 & 2.31 & 0.00 & 4.03 \\
\hline & Núm. ponderado & 5 & 7 & 0 & 12 \\
\hline & $\%$ ponderado & 1.66 & 2.33 & 0 & 3.99 \\
\hline \multirow[t]{4}{*}{ Total } & Núm. absoluto & 65 & 241 & 41 & 347 \\
\hline & $\%$ absoluto & 18.72 & 69.46 & 11.82 & 100.00 \\
\hline & Núm. ponderado & 60.5 & 202.5 & 38 & 301 \\
\hline & $\%$ ponderado & 20.10 & 67.27 & 12.63 & 100.00 \\
\hline
\end{tabular}

Tabla 3. Tabla de contingencia de facetas bajo las que son tratados los procesos de gestión documental en la LGA 
La segunda faceta con mayor porcentaje de valor ponderado es Actividades, con $20.10 \%$. Las categorías tratadas bajo esta faceta aluden directamente a las acciones o conjuntos de acciones que integran la actividad, las cuales varían dependiendo de cada categoría.

La faceta Elementos presenta los valores más bajos, con un porcentaje de $12.63 \%$ sobre el número ponderado de referencias. En todas las categorías temáticas, salvo en Acceso y transparencia, esta faceta se encuentra en último lugar.

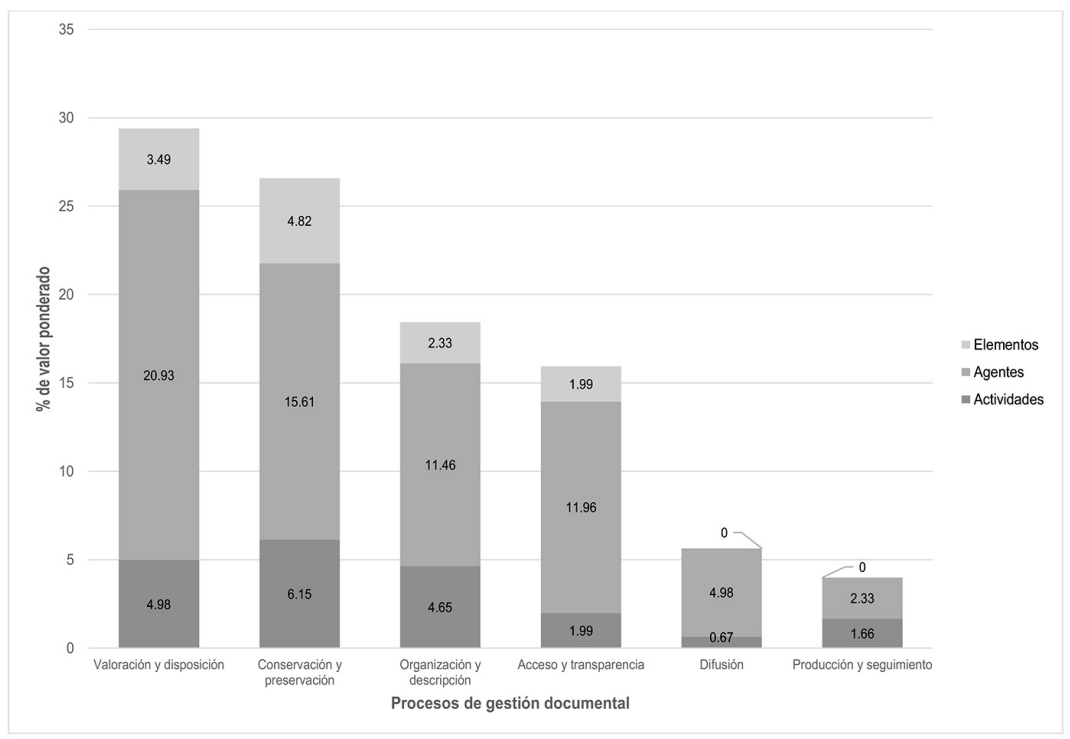

Figura 2. Facetas bajo las que son tratados los procesos de gestión documental en la LGA

\section{Discusión}

El análisis de los resultados obtenidos permite advertir el elevado grado de coincidencia de la LGA con fuentes normativas de carácter técnico como UNE-ISO 15489-1 (2016) o UNE-ISO 30301 (2011), ya que la mayoría de los procesos relacionados en esas normas son tratados en la Ley: creación, captura, clasificación, acceso, almacenamiento, migración, conversión y disposición. Los únicos procesos a los que se alude en las normas que no están presentes en la Ley son los de uso y reutilización de la información e indización (UNE-ISO 15489-1, 2016). Asimismo, los procesos recogidos en la Ley coinciden con los definidos por otros autores; en algunos casos completamente (Bustelo Ruesta, 2017; Villanueva Bazán et al., 2002) y en otros en un nivel muy elevado (Bustos Pretel, 2018). 
Es posible diferenciar cuatro tipos de disposiciones sobre cada proceso: disposiciones con definiciones de conceptos relacionados con los procesos, disposiciones de carácter programático o declarativo, disposiciones sobre obligaciones de los agentes implicados, y disposiciones técnicas. De todas ellas, son las últimas las que resultan más relevantes para la gestión de los procesos, ya que proporcionan pautas detalladas para su desarrollo.

La Ley incluye en su articulado no sólo referencias a procesos tradicionales (valoración, organización, conservación...), sino también a algunos procesos relacionados con las fases iniciales de generación y uso de los documentos, tales como producción, trazabilidad, interoperabilidad o seguridad de información. La presencia de estas referencias es significativa, porque promueve la armonización de la Ley con normas complementarias sobre administración digital (García González, 2018; Cerrillo Martínez, 2017; Bustelo Ruesta y García Morales, 2008), interoperabilidad (Interoperabilidad, 2016), transparencia y valores democráticos (Aguilera Murguía, 2017). En definitiva, tal como señala el profesor Alberch i Fugueras (2013: 24), es preciso tender a la complementariedad legislativa.

\section{Consideraciones finales}

Desde el punto de vista cuantitativo, existe un predominio claro de tres categorías tradicionales: Valoración y disposición, Conservación y Organización y descripción (más de 74 \% de las referencias identificadas corresponden a estas tres categorías).

De forma complementaria, cabe señalar que la Ley General de Archivos también presta atención a los procesos relacionados con las fases de creación, captura y gestión inicial de los documentos, ya que se advierte que el número de referencias sobre Producción y seguimiento, Valoración y disposición y Acceso y transparencia se encuentra próximo a 50 \% del total (46.98\%). A esta cantidad habría que sumar los valores de otras subcategorías, como Preservación digital o Descripción/gestión de metadatos, que se encuentran directamente vinculadas con el tratamiento de los documentos en estas primeras fases. De este modo, es posible concluir que en la redacción de la ley se han sabido conjugar los procesos archivísticos tradicionales con otros de carácter más innovador.

Aunque la Ley General de Archivos destaca por la atribución de numerosas competencias y obligaciones a los agentes implicados en su desarrollo, también lo hace por la presencia de numerosas disposiciones sobre aspectos técnicos (planificación del proceso de conservación, conservación de documentos 
digitales, niveles del cuadro de clasificación, conservación de metadatos, criterios de valoración, elaboración de instrumentos, etc.).

Resulta también relevante la existencia de un discreto pero consistente número de referencias sobre difusión de documentos y archivos, el cual parece destacar la importancia del trabajo de divulgación archivística.

Es posible afirmar, asimismo, que la LGA es un texto legal novedoso en el ámbito de los archivos, por incluir referencias específicas sobre conceptos como trazabilidad, interoperabilidad, seguridad de la información, metadatos y numerosas referencias a documentos electrónicos. Conceptos que ya se encuentran en la realidad del presente y que marcarán el futuro de la disciplina.

\section{ReFERENCIAS}

Aguilera Murguía, Ramón. 2017. Gestión de documentos y nuevos valores democráticos. Evolución e involución de los archivos públicos mexicanos. Ciudad de México: Centro de Investigación y Docencia Económicas.

Alberch i Fugueras, Ramón. 2013. Archivos: entender el pasado, construir el futuro. Barcelona: Universitat Oberta de Catalunya.

Bardin, Laurence. 1991. Análisis de contenido. Madrid: Akal.

Barnard Amozorrutia, Alicia. 2011. "Funciones, procesos y requisitos", en Administración de documentos y archivos: textos fundamentales, coordinado por José Ramón Cruz Mundet, 67-116. Madrid: Coordinadora de Asociaciones de Archiveros. http://hdl.handle.net/10016/19730

Bustelo Ruesta, Carlota. 2011. "Los grandes temas relacionados con la gestión de documentos: desafíos y oportunidades”. El profesional de la información 20 (2): 129-133. https://doi.org/10.3145/epi.2011.mar.01

Bustelo Ruesta, Carlota. 2017. Procesos de creación y control de documentos. Barcelona: Universitat Oberta de Catalunya.

Bustelo Ruesta, Carlota y Elisa García Morales. 2008. "Administración electrónica y gestión documental. Consideraciones a la luz de la Ley para el Acceso Electrónico de los Ciudadanos a los Servicios Públicos”. El profesional de la información 17 (1): 106-111. https://doi.org/10.3145/epi.2008.ene.13

Bustos Pretel, Gerardo (coord.). 2018. La gestión del documento electrónico. Las Rozas: Wolters Kluwer.

Cerrillo Martínez, Agustí (coord.). 2017. A las puertas de la administración digital. Una guia detallada para la aplicación de las Leyes 39/2015 y 40/2015. Madrid: Instituto Nacional de Administración Pública.

Daines, J. Gordon. 2011. "Re-engineering archives: Business Process Management (BPM) and the quest for archival efficiency". The American archivist (74): 123-157 https://doi.org/10.17723/aarc.74.1.h8159344u8331165

Evans, Frank B. 1990. "Records and administrative processes: retrospect and prospects”. Trabajo presentado en International Council on Archives' symposium on current records. Otawa, 15-17 de mayo. 
Gallardo Taboada, José Ricardo. 1987. "Un lugar para cada documento y cada documento en su lugar: la archivística y sus procesos técnicos". ALA: órgano de comunicación de la Asociación Latinoamericana de Archiveros (México, Asociación Latinoamericana de Archivos) (3): 9-17.

http://www.mcu.es/ccbae/es/catalogo_imagenes/grupo.cmd?path=9488\&texto_busqueda $=$

García González, María. 2018. e-Administración, realidad encontrada tras la aplicación de un método descriptivo de gestión de procesos administrativos en entidades locales españolas. Madrid: Congreso de los Diputados, Departamento de Publicaciones.

Interoperabilidad y documento electrónico. 2016. Madrid: Ministerio de Economía y Hacienda, Ministerio de Economía y Competitividad.

ISO 9001. 2015. Sistemas de gestión de la calidad - Requisitos. Ginebra: ISO.

LGA (Ley General de Archivos). 2019. Nueva ley DOF 15-06-2018. México: Cámara de Diputados del H. Congreso de la Unión.

Llansó Sanjuan, Joaquim. 2012. "La integración de la gestión de documentos y la administración de archivos entre los procesos transversales de las organizaciones. El caso de la Universidad Pública de Navarra”. Tría (18): 63-72. https://www.archiverosdeandalucia.org/wp-content/uploads/2019/04/Numero_18_006.pdf

Pérez Fernández de Velasco, José Antonio. 2007. Gestión por procesos. Madrid: ESIC.

Sainz Varela, José Antonio. 2011. "La gestión de recursos en un sistema de archivo", en Administración de documentos y archivos: textos fundamentales, coordinado por José Ramón Cruz Mundet, 311-376. Madrid: Coordinadora de Asociaciones de Archiveros. http://hdl.handle.net/10016/19730

UNE-ISO 15489-1. 2016. Información y Documentación, Gestión de documentos. Parte 1: conceptos y principios. Madrid: AENOR.

UNE-ISO 30301. 2011. Información y Documentación, Sistemas de gestión para los documentos. Requisitos. Madrid: AENOR.

UNE-ISO/TR 26122 IN. 2008. Información y documentación. Análisis de los procesos de trabajo para la gestión de documentos. Madrid: AENOR.

Villanueva Bazán, Gustavo, Georgina Flores Padilla, Roberto Montores Martínez, Martha O. Ochoa Esquivel y Luis Torres Monroy. 2002. Manual de procedimientos técnicos para archivos históricos de universidades instituciones de educación superior. Puebla: Benemérita Universidad Autónoma; Ciudad de México: Universidad Autónoma de México. https://issuu.com/oscarn/docs/archivos

Wimmer, Roger D. y Joseph R. Dominick. 1996. La investigación cientifica de los medios de comunicación: una introducción a sus métodos. Barcelona: Bosch.

Zaratiegui, José Ramón. 1999. "La gestión por procesos: su papel e importancia en la empresa”. Economía industrial (330): 81-88.

https://www.mincotur.gob.es/Publicaciones/Publicacionesperiodicas/EconomiaIndustrial/RevistaEconomiaIndustrial/330/12jrza.pdf 


\section{Para citar este texto:}

Bonal-Zazo, José-Luis y María-Pilar Ortego-de-Lorenzo-Cáceres. 2020.

"Los procesos de gestión de documentos en la Ley General de Archivos de México: análisis de contenido desde una perspectiva cuantitativa". Investigación Bibliotecológica: archivonomía, bibliotecología e información 34 (85): 179-197.

http://dx.doi.org/10.22201/iibi.24488321xe.2020.85.58232 